\title{
ParaDyn A Parallel
} Nonlinear, Explicit, ThreeDimensional FiniteElement Code for Solid and Structural Mechanics User Manual

\author{
C. G. Hoover, A. J. De Groot, R. J. Sherwood
}

\section{June 1, 2000}

U.S. Department of Energy

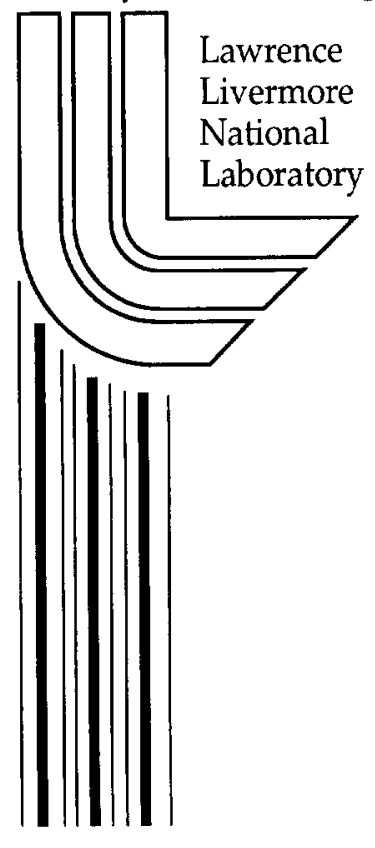




\section{DISCLAIMER}

This document was prepared as an account of work sponsored by an agency of the United States Government. Neither the United States Government nor the University of California nor any of their employees, makes any warranty, express or implied, or assumes any legal liability or responsibility for the accuracy, completeness, or usefulness of any information, apparatus, product, or process disclosed, or represents that its use would not infringe privately owned rights. Reference herein to any specific commercial product, process, or service by trade name, trademark, manufacturer, or otherwise, does not necessarily constitute or imply its endorsement, recommendation, or favoring by the United States Government or the University of California. The views and opinions of authors expressed herein do not necessarily state or reflect those of the United States Government or the University of California, and shall not be used for advertising or product endorsement purposes.

This work was performed under the auspices of the U. S. Department of Energy by the University of California, Lawrence Livermore National Laboratory under Contract No. W-7405-Eng-48. 


\section{ParaDyn}

\section{A Parallel Nonlinear, Explicit, Three-Dimensional Finite-Element Code for Solid and Structural Mechanics}

\section{User Manual}

Carol G. Hoover, Anthony J. De Groot, Robert J. Sherwood

\section{Methods Development Group} Mechanical Engineering

ParaDyn Version 1.01

June 2000 


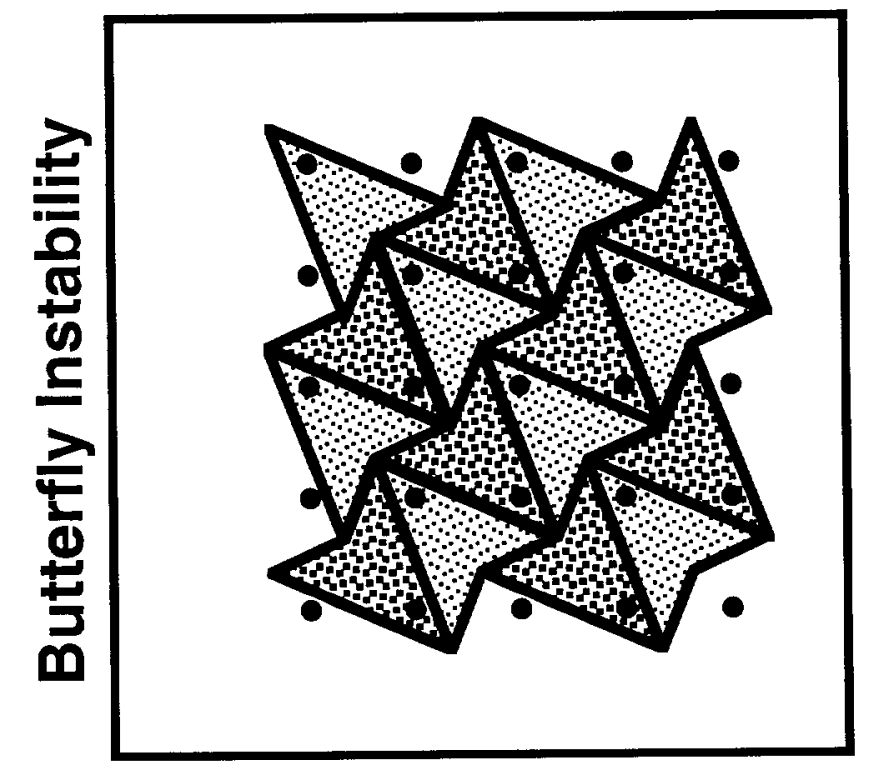




\section{Preface}

This User Manual documents the collaborative code development work with my colleagues, Tony De Groot and Bob Sherwood. Our efforts represent the majority of the original work in the parallel algorithm design and development for the ParaDyn Project. Doug Speck, Elsie Pierce, and Vic Castillo are now substantive contributors to the ParaDyn Project. Doug and Elsie, in particular, have made it possible to quickly design flexible binary databases (MILI) and have developed significant enhancements to the GRIZ visualization software. Their work paves the way for the visualization capabilities needed when using parallel computers with hundreds and thousands of processors.

Analysts provide the insights needed to turn our software development into an effective production tool for finite-element engineering analysis. At the Lawrence Livermore National Laboratory (LLNL) Dan Badders, Tony Lee, and Tony DePiero entered the turbulent waters of massively parallel computers earliest and have provided very valued input to our code development efforts.

Raju Namburu and Photios Papados are collaborators from the Army Research Laboratory (ARL) and the Engineer Research and Development Center (ERDC). They led the way to the first multimillion element calculations on Department of Defense high performance computers. John Benner and colleagues from the Los Alamos National Laboratory (LANL) were the first to use the ParaDyn program on over 1000 processors.

We especially appreciate the very valuable comments and support that our collaborators from ARL, ERDC, and LANL have provided for our code development efforts.

Carol Hoover

Methods Development Group

Mechanical Engineering Department

Lawrence Livermore National Laboratory

Livermore, California 94551-7808

925-422-1556 hoover1@Ilnl.gov 


\section{Abstract}

ParaDyn is a parallel version of the DYNA3D computer program, a three-dimensional explicit finite-element program for analyzing the dynamic response of solids and structures. The ParaDyn program has been used as a production tool for over three years for analyzing problems which range in size from a few tens of thousands of elements to between one-million and ten-million elements. ParaDyn runs on parallel computers provided by the Department of Energy Accelerated Strategic Computing Initiative (ASCI) and the Department of Defense High Performance Computing and Modernization Program. Preprocessing and post-processing software utilities and tools are designed to facilitate the generation of partitioned domains for processors on a massively parallel computer and the visualization of both resultant data and boundary data generated in a parallel simulation. This manual provides a brief overview of the parallel implementation; describes techniques for running the ParaDyn program, tools and utilities; and provides examples of parallel simulations. 


\section{TABLE OF CONTENTS}

PREFACE

ABSTRACT ii

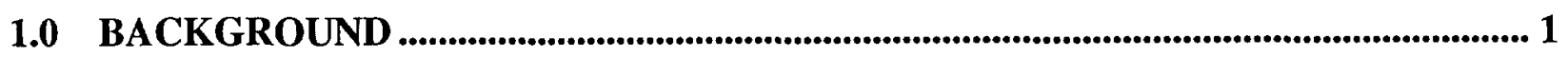

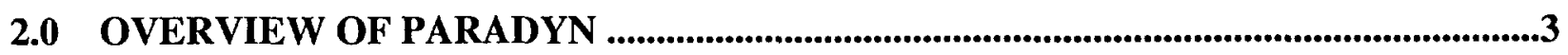

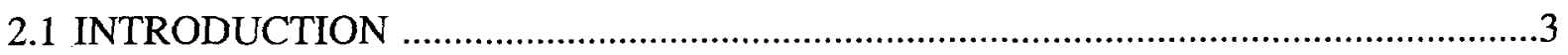

2.2 THE PARALLEL FINITE-ELEMENT MODEL ................................................. 4

2.3 PARALLEL PERFORMANCE AND SCALABILITY ........................................... 8

2.4 SCALABLE PARALLEL CONTACT ALGORITHMS .......................................... 9 PARALLEL LOCAL CONTACT ............................................................ 11

PARALLEL AUTOMATIC CONTACT …................................................. 12

CONCLUDING REMARKS ON PARALLEL CONTACT.............................. 13

2.5 BOUNDARY CONDITIONS AND CONSTRAINTS ........................................... 13

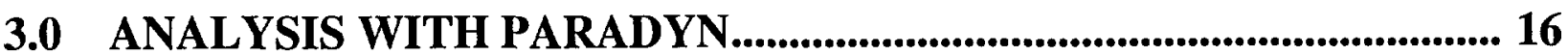

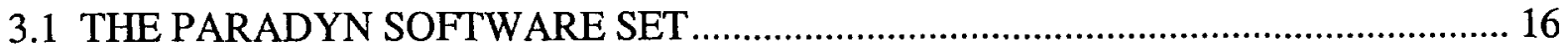

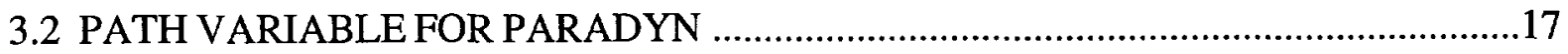

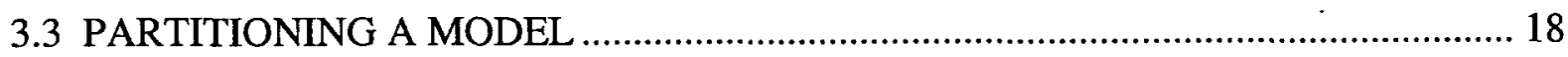

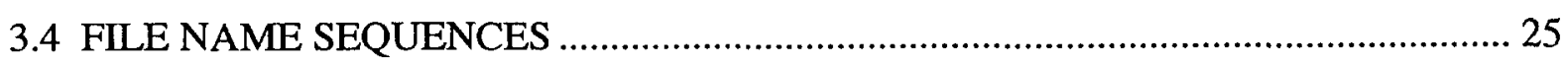

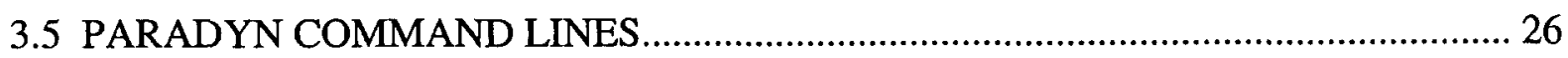

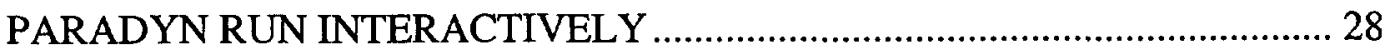

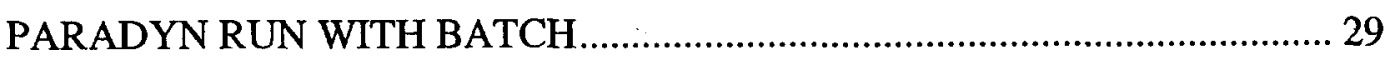

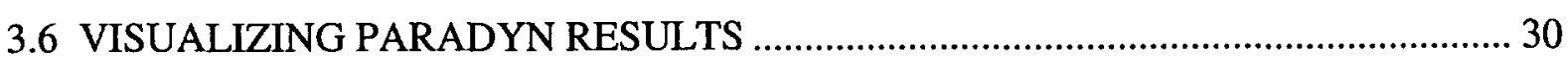

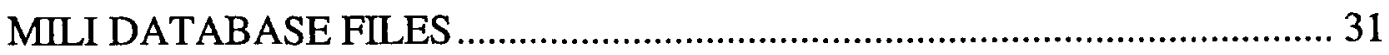

COMBINING PARALLEL DATABASES .............................................. 32

3.7 SUMMARIZED STEPS FOR USING PARADYN............................................ 33

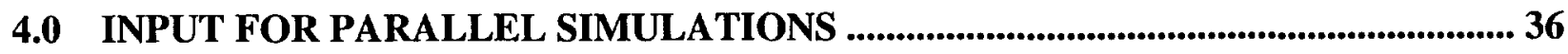

4.1 STATIC INITIALIZATION AND DYNAMIC ANALYSIS ................................... 36

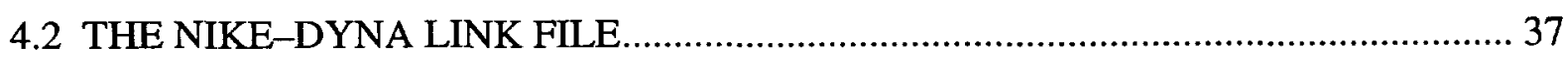

4.3 MULTIPLE VERSIONS OF RUNNING RESTART FILES ....................................38

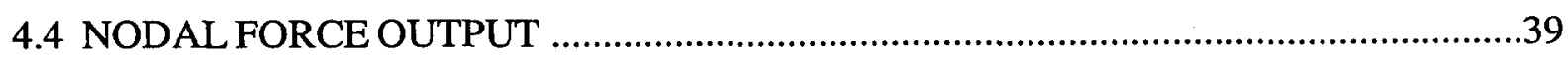

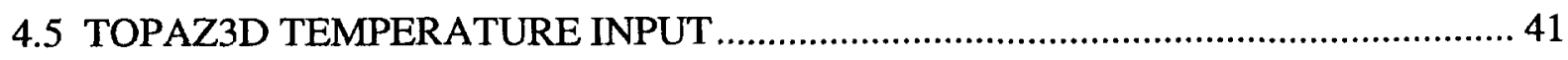




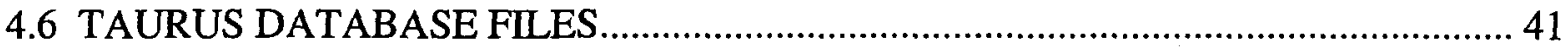

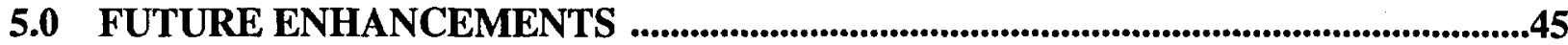

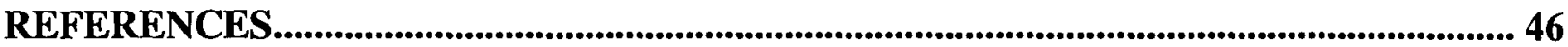




\subsection{BACKGROUND}

Significant speed gains (for example, factors of sixty or more on sixty-four processors) are being achieved for engineering design calculations on parallel computers with as few as a hundred processors using ParaDyn, the parallel version of the DYNA3D program [1]. The latest massively parallel computers with thousands of processors now make it possible to design engineering models for mechanical system analysis with multiple component parts as well as to add more detail and complexity in the models for components. ParaDyn and DYNA3D are explicit finite-element programs designed to solve for the nonlinear, transient response of solids and structures. The two programs, contained within a single source, are developed by the Methods Development Group at the Lawrence Livermore National Laboratory (LLNL). The web site, http://www.llnl.gov/eng/ mdg/mdg_home.html, provides general information and online documentation about the complete family of themomechanical programs developed in the Methods Development Group.

Parallel computers are now commonplace in our computing environment. Computers with speeds ranging from 1 to 100 TeraOps $\left(10^{12}\right.$ to $10^{14}$ operations per second) and thousands of processors will be delivered through the year 2004 to the Accelerated Strategic Computing Initiative (ASCI) Program. These computers dominate the high-end computing at LLNL. More modest parallel workstation clusters with ten or twelve processors support the Institutional Programs at LLNL. ParaDyn is implemented using the Message Passing Interface (MPI) standard. This standard makes it possible to develop parallel engineering applications that run on both distributed memory massively parallel computers and also shared memory workstation clusters. A summary of the computer resources at LLNL may be viewed at http://www.llnl.gov/asci/platforms and http:// www.llnl.gov/sccd/FAST.resources.html.

The Department of Defense High Performance Computing and Modernization Program is also a very strong participant in acquiring hardware and developing software for next-generation massively parallel computers. Computer characteristics in terms of size, speed, and number of processors at the DOD Major Shared Resource Centers (MSRCs) are summarized in the links provided by the Program Office web site at http://www.hpcmo.hpc.mil. These data illustrate the continuing significant increase in our parallel computational speeds and capacities.

ParaDyn is a parallel production program. Code development efforts are now directed toward optimizing the parallel algorithms, developing parallel preprocessing and post-processing software, and developing software tools for engineering optimization studies. Concurrent with the 
parallel development effort, many code developers for the DYNA3D program are contributing new algorithms, elements, and material models to enhance the mechanics modeling capabilities. A single source encompassing both the ParaDyn and DYNA3D algorithms makes it possible to naturally migrate the DYNA3D enhancements into the production ParaDyn program. Finally, coupled programs for thermal, mechanical, and fluid analysis are being designed for parallel computers. A production capability for coupled analysis is possible in the next few years. 


\subsection{OVERVIEW OF PARADYN}

\section{$2.1 \quad$ Introduction}

Advances in the development of parallel algorithms for explicit finite-element analysis and domain partitioning techniques have led to scalable production applications using ParaDyn. This has resulted in several benefits to our engineering design programs. Firstly, calculations are now performed in a day or less for problems that previously ran over several weeks. Secondly, new models are being generated for mesh sizes between one-million and ten-million elements. This is an order of magnitude larger than the largest models possible in the past. Finally, longer time simulations (problems running for a few million steps) for small to moderate sized problems are now being run on both massively parallel computers and workstation clusters.

Analysts play an important new role in preparing models for parallel computers. The meshes are increasingly much larger and more complex. New validation tools are needed for the mesh generation step, specification of boundary loads and constraints, and defining facets on interfaces. In addition, the modeling of contact interfaces can have a significant effect on the parallel performance and scalability. Optimizing the performance and achieving scalability of parallel contact algorithms is particularly challenging. Two distinct forms of parallel contact algorithms have been developed in ParaDyn and will be discussed in the sections on parallel contact.

Current and future parallel algorithm development is focused on contact algorithm research and also on providing the necessary automated modeling tools for the analyst. The development of the GRIZ4 visualization tool [2] based on the new MILI (Mesh I/O Library) software [3] is one step toward achieving this goal. In addition, we anticipate further research in numerical methods to enhance the scalability of parallel contact algorithms.

This manual is available in PDF form for interactive viewing. In some sections the text is displayed in either red or blue. The blue highlighting indicates text passages that are included in a version of the manual designed for a specific computer or site location. Thus, the blue text indicates conditional text which has been generated with the particular version of the manual being viewed. Red text is used to highlight cautionary notes. For instance, red text is used to highlight a warning message to copy output files between ParaDyn runs when those files would otherwise be overwritten by a subsequent run in the same directory. 
To prepare input data and select control options and flags for the ParaDyn program, follow the discussions in the DYNA3D User Manual [1]. An online manual for DYNA3D is available on the home page for the Methods Development Group, http://www.llnl.gov/eng/mdg/mdg_home.html. Instructions for using the new preprocessing and post-processing software for parallel simulations are included in this manual. In this manual the term preprocessing refers to the software used after the mesh generation step to prepare the model for the analysis with the ParaDyn program. More specifically it refers to the partitioning of the model. Computer-specific instructions and example problems are provided here to illustrate how to run a ParaDyn analysis on a parallel computer. Further documentation for the preprocessing software for partitioning meshes for ParaDyn is available online with the ParaDyn software. This documentation plus installation instructions are also available in an LLNL UCRL Report [4]. And finally, some standard features in DYNA3D requiring additional documentation for a parallel analysis are included in this manual.

A set of typeface conventions is followed throughout this manual to allow the reader to easily distinguish between commands, parameters, and computer generated text. Commands that appear in bold type should be entered verbatim. Parameters that appear in italic type should be given values when included in the input. Computer generated text, such as error messages or default file names, is printed in a typewriter-like (Courier) font. In text passages file names appear in italic type for clarity.

The next sections provide introductory discussions about parallel algorithms and computers. Section 2.2 discusses the parallel finite-element model and describes partitioning methods. Section 2.3 discusses parallel performance and scalability measurements. Section 2.4 characterizes contact interfaces and their implementation in parallel. This section concludes with guidelines for modeling contact interfaces with parallel algorithms.

\subsection{The Parallel Finite-Element Model}

A successful strategy for parallel implementation of the explicit finite-element method is based on dividing the mesh among the processors and executing ParaDyn on a subdomain in each processor [5]. The elements from the mesh are divided into subdomains so that each processor has approximately the same amount of calculations to perform in a timestep. The nodes on the boundaries of a subdomain are referred to as shared nodes. Nodal force data for shared nodes are communicated between processors when the nodal force updates are calculated. The nodal points 
on the subdomain boundaries may be duplicated (shared) in more than one processor. Mesh partitioning is the strategy for dividing the problem into subdomains and mapping subdomains to processors. This is illustrated for two processors in Figure 1.

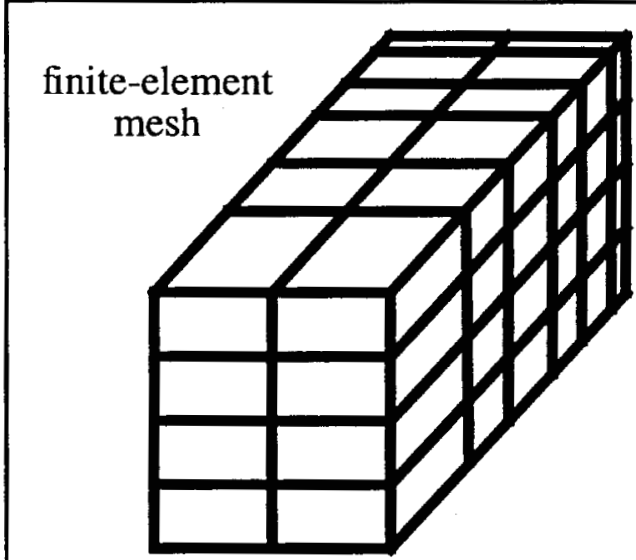

(a)

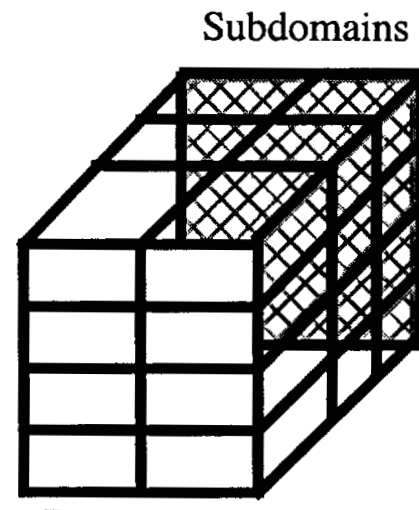

Processor 0

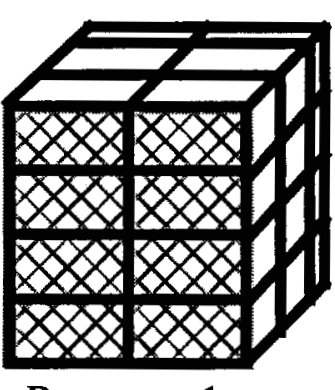

Processor 1

Figure 1. Two subdomains on a finite-element mesh. (a) The original mesh with 48 elements is partitioned into two subdomains with 24 elements each. (b) The calculations involving nodal points on the cut plane (shown as patterned) are performed in both processors. The 15 nodes on the cut plane are referred to as shared nodes.

The nodal forces consist of contributions from applied loads, contact interactions, and internal deformations.

$F_{\text {node }}=F_{\text {applied }}+F_{\text {contact }}-F_{\text {internal }}$

The internal force calculation for a shared node includes a contribution from elements in different processors. Each processor calculates a partial nodal force for the elements in its processor. These partial force contributions are communicated between the processors so that the total force computed for a shared node is the same in all processors within the error introduced by the ordering of the calculations. As much as possible, the ParaDyn algorithms are designed to store partial nodal force data for shared nodes until all contributions to the nodal force have been computed before communicating the shared data.

Research in applied mathematics has led to efficient techniques for subdividing or partitioning the complicated unstructured meshes that arise in practical engineering applications [6-9]. We use the METIS software from the University of Minnesota to partition finite-element meshes and contact 
surfaces. (For more information on METIS, see http://www-users.cs.umn.edu/ karypis/metis/ main.shtml). The METIS algorithms use a graph to represent the finite-element-mesh.

Preprocessing software automatically produces the graphs needed for the mesh partitioning step.

Mesh partitioning is accomplished by representing a finite-element mesh as a graph. A graph has vertices and interconnecting edges. The vertices and edges represent objects on the mesh. For finite-element meshes, the vertices of the graph correspond to elements (zones) in the mesh and the edges correspond to nodes in common between two connected elements. This is illustrated in Figure 2.
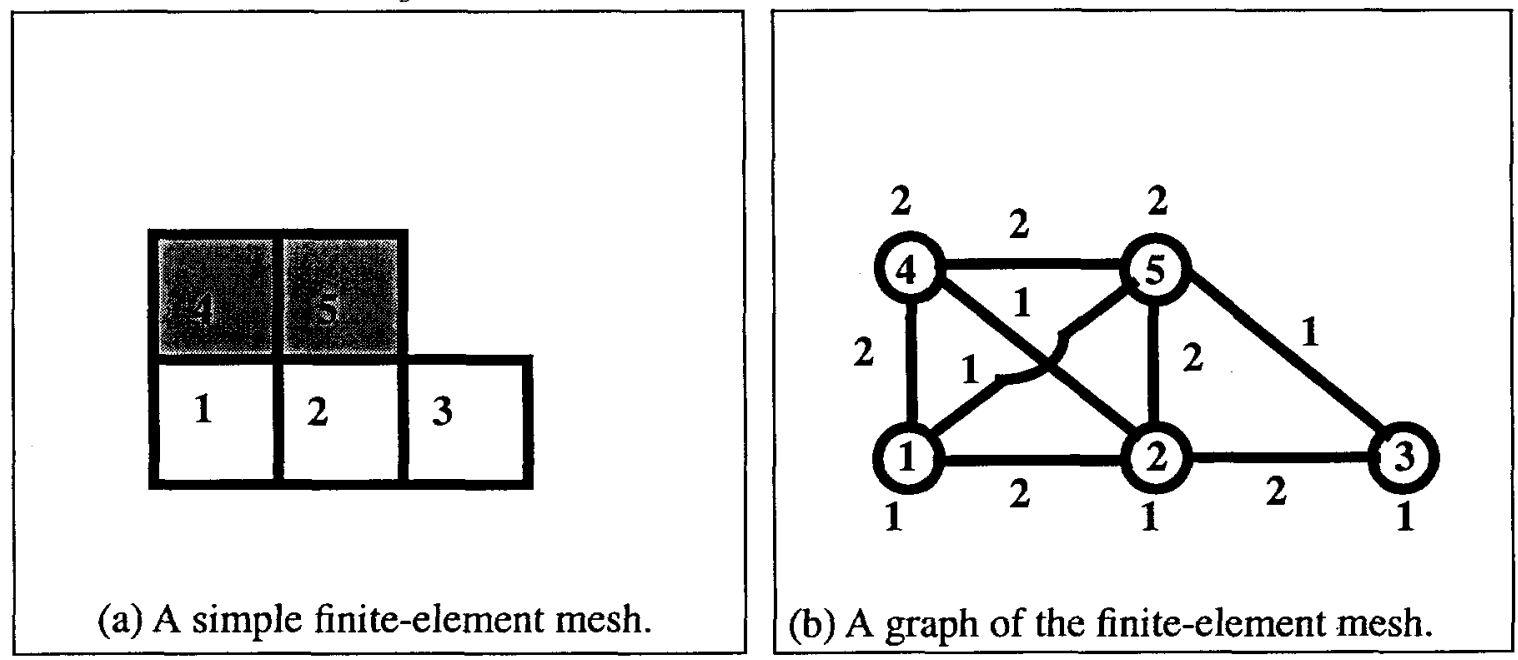

Figure 2. A finite-element mesh and the graph representation of the mesh. (a) This simple mesh consists of two materials, shaded and unshaded. The shaded material requires twice as much calculation time as the unshaded material. (b) This is the graph of the mesh. The vertices are represented as circles and the number near a vertex is the computational weight of the vertex. The lines connecting the vertices are edges and represent the shared data between the vertices. The number specified along the edges represents the number of shared nodes between the two elements represented by the vertices.

The graph represents the element-to-element connectivity for the mesh. The METIS algorithms find an optimal division of the graph corresponding to a specified number of subdomains. An important aspect of graph partitioning techniques is the use of weighting factors for both the vertices and edges. These weighting factors are used to balance the vertices into sets of roughly equal weight and thus, provide intelligent input control over the partitioning of the mesh. To illustrate this, the relative computational cost for a complex material model, a boundary condition or any other expensive part of the calculation, can be used to weight the vertices. Similarly, an edge 
in the graph represents the number of common nodes between the elements and can be appropriately weighted by a relative measure of the shared data communicated if the graph is cut on that edge. Figure $2 b$ indicates the edge weights used for the few elements shown in Figure $2 a$.

The partitioning task is automated completely in the preprocessing tool, DYNAPART, for any mesh geometry. This software was used for the assignment of a one-million element mesh to 128 processors as shown in Figure 3. The colors are used to show the processor assignment for subdomains on the mesh. The mesh was developed to model a shock moving from the top vertex of the mesh in the direction of the half-cylindrical cavity region located half way down and on the left-hand side of the mesh. The mesh is zoned very finely at the top of the model to resolve the shock structure. As a result of this fine zoning the subdomains are much smaller at the top of the mesh than the subdomains on the lower part of the mesh where the zoning is coarse. The mesh lines are not shown in the Figure.

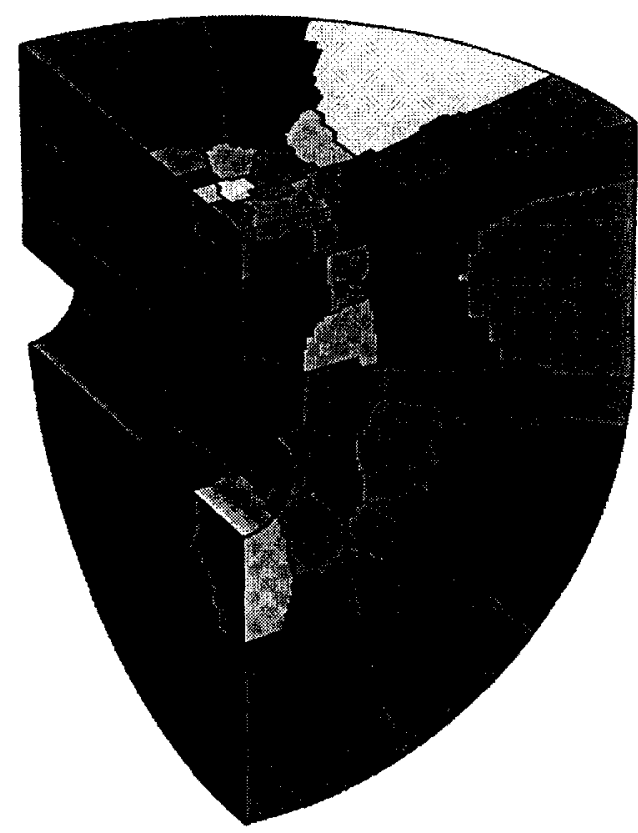

Figure 3. Processor assignment for a one-million element mesh. Colors are used to distinguish the subdomains assigned to 128 processors. This problem without sliding interfaces scales linearly as the number of processors is increased up to roughly 1000 processors. 


\subsection{Parallel Performance and Scalability}

The time required to deliver results on a parallel computer is the sum of the time for computing results on the processors and the time for communicating data between the processors.

where

$$
\tau_{w c}=\tau_{c a l c}+\tau_{c o m m}
$$

$\tau_{w c}$ is the total elapsed time taken for the calculation (total wall clock time);

$\tau_{\text {calc }}$ is the elapsed time the processors spend computing results;

$\tau_{\text {comm }}$ is the elapsed time the processors spend communicating shared data.

Ideally, if the number of processors is doubled, the rate for delivering results will be doubled. In practice this linear scaling of the delivery rate with the number of processors breaks down when the communication time becomes significant compared to the amount of time processors spend computing results.

If the parallel calculation is efficient, the delivery time $\tau_{w c}$, for $\mathrm{N}_{p}$ processors will be approximately equal to $1 / \mathrm{N}_{p}$ of the time for calculating the same results on one processor, $\tau_{1}$. Thus, a measure of the parallel efficiency is given by the following:

$$
\varepsilon=\tau_{1} /\left(\mathrm{N}_{p} \tau_{w c}\right) \times 100 \%
$$

The preprocessing software tools in DYNAPART provide convenient methods for selecting the maximum optimal number of processors to use for a ParaDyn simulation. Using more processors will result in no further improvement in delivering results and wastes computing resources.

The optimal number of processors to select for a parallel calculation without contact can be estimated by specifying the minimum number of elements that can be allocated to a processor, which results in a negligible amount of communication time (ideally less than $10 \%$ ) compared to the computation time for the deformation calculations. The statistics calculated by the partitioning software provide quantitative values for determining the optimal number of processors. These statistics will be discussed in the section describing the partitioning software tools. For current models, an estimate for the minimum number of elements to assign to a processor is between 1000 and 2000 .

Almost all problems of interest include contact interface definitions, which are always more difficult to run efficiently in parallel. 


\subsection{Scalable Parallel Contact Algorithms}

Designing efficient parallel contact algorithms is challenging because the surface motion is often unpredictable and new interfaces may appear dynamically. As a result, dividing the problem domain into subdomains that are optimal for calculating the element deformations will almost never result in an efficient division of the problem for the contact calculations. Hence, the ParaDyn software uses partitioning methods for the sliding interfaces that differ from the partitioning for the mesh. The results for the contact force calculations are communicated once in a timestep to the processors defined by the mesh partition. Similarly the nodal position and velocity updates are communicated from the processors defined by the mesh partition to the processors defined by the contact partitions once in a timestep.

There are currently two parallel contact algorithms in the ParaDyn program [10] and selecting the algorithm to use will depend on the predictability of the surface motion as well as the relative size of the largest interfaces. In some problems, surfaces initially close together engage in small relative motion and the contact remains in a localized region of the mesh. We refer to this as local contact. Sliding interface types (1-3, 5-10) in DYNA3D/ParaDyn are local algorithms. For other problems with large deformation or moving parts, the motion of the surfaces is not predictable. Thus, more sophisticated and computationally expensive searches for the surfaces in contact must be performed throughout the simulation. We refer to this as arbitrary contact. Examples illustrating arbitrary contact are a ball rolling on a plane, a surface folding on itself, or an automobile crash simulation. Arbitrary contact is implemented in DYNA3D automatic contact interfaces, types 12 through 14 . The automatic contact algorithm type 14 is the material erosion algorithm (SAND).

The parallel algorithm implementing local contact allocates a contact interface (both the master surface and the slave segments or nodes) into one processor. This method is very efficient and useful for problems with many contact surfaces that are relatively small. The method can limit the scalability of the problem if there are large contact surfaces because the partitioning for contact may require more elements in a processor than is efficient for an optimal mesh partitioning. In this latter case, the problem can often be made more scalable by using the parallel automatic contact algorithms described below.

The second parallel contact algorithm models arbitrary interface contact. The search for arbitrary contact is implemented in the DYNA3D automatic contact algorithms. The method uses a set of cells (buckets) for sorting and grouping surface nodes and segments into localized regions on the mesh. The parallel version of the algorithm partitions the buckets among the processors to balance 
the contact calculations among them and minimize the communication of nodes and surface faces in cells with data that must be shared between processors. An additional step is needed in the automatic contact algorithm when contact surface motion requires a regeneration of the cells and sorting of the surfaces into the new cells. The frequency of the bucket-regeneration step is automatically computed or, in some unusual situations, can be specified as a user input. In ParaDyn the bucket-regeneration step induces extra communication during the timestep over which it occurs. Thus, it is important to rebucket as infrequently as possible to avoid the extra communication costs.

The arbitrary contact algorithms are referred to as automatic contact algorithms in the DYNA3D documentation. The reason for this is that the faces on the contact surfaces are generated automatically in DYNA3D and ParaDyn. This is in contrast to the older more tedious method in which the contact interfaces are defined by the analyst when generating the mesh. For the large meshes generated for parallel simulations this is an extremely time consuming task. Furthermore, the contact interface definition is often a source of errors in the input file because of difficulties encountered during the part-merging phase of the mesh generation.

The parallel contact algorithm in ParaDyn is selected by the DYNA3D sliding interface method used for modeling the contact. Sliding-interface types (1-3, 5-10) are implemented by assigning each contact interface into a processor. Sliding interface type 11 (SAND) is not implemented in parallel because the equivalent interface is modeled in the more robust and newer automatic contact interface type 14 . The single surface contact algorithm in type 4 is implemented as a full surface assigned to a processor. A more general version of single surface contact is contained within interface types 12 or 13 .

Sliding interface types 12 and 13 are identical in their parallel implementation. However, type 13 provides options for controlling the search and other features as follows:

Boxes can be defined to limit the domain of the search;

Material can be included or excluded in the boxes for the search;

Faces defined as in a type 3 interface can be specified rather than automatically generated.

See the DYNA3D user manual in the keyword options section for a description of the input for sliding interface type 13 . 
The most commonly used sliding interface in DYNA3D is type 3 . The same physical interface condition (sliding with friction and voids) can also be modeled with the automatic contact algorithm types 12 and13. The parallel efficiency for either choice for the sliding interface may vary significantly depending on the details of the interfaces and the size of the model. The next sections compare the two forms of parallel contact algorithms. These comments provide guidelines for selecting which algorithm to use for an efficient parallel calculation.

\subsubsection{Parallel Local Contact}

The parallel local algorithm allocates each sliding interface fully in one processor. For more than one sliding interface, the DYNAPART preprocessing software distributes the set of interfaces among as many processors as possible. Special graph weighting methods are used for partitioning the contact interfaces and evenly distributing the contact calculations among processors. This algorithm is illustrated with the spin forming mesh shown in Figure 4. This model consists of a rotating plate formed into a hemispherical shape by rollers in contact with the plate surface. A four-processor partition for this problem cuts the plate into three concentric rings. The center ring and brushes form the sliding interface and are fully contained in one processor. An eight-processor partition for the problem cuts two of the rings in half. The sliding interface, the center ring and the rollers, remains uncut.

The number of processors used for problems with local contact is often limited by the largest sliding interface. If the surfaces on the largest interface contain many more elements than the minimum number of elements that will provide a balanced calculation for the element deformation, then the optimal number of processors for the calculation will be determined by the contact calculations. A more subtle condition for large contact surfaces occurs when the partitioning cuts the mesh one element below the surfaces in the sliding interface. This results in a large amount of shared-node communication for processors with elements connected to the contact surfaces. 

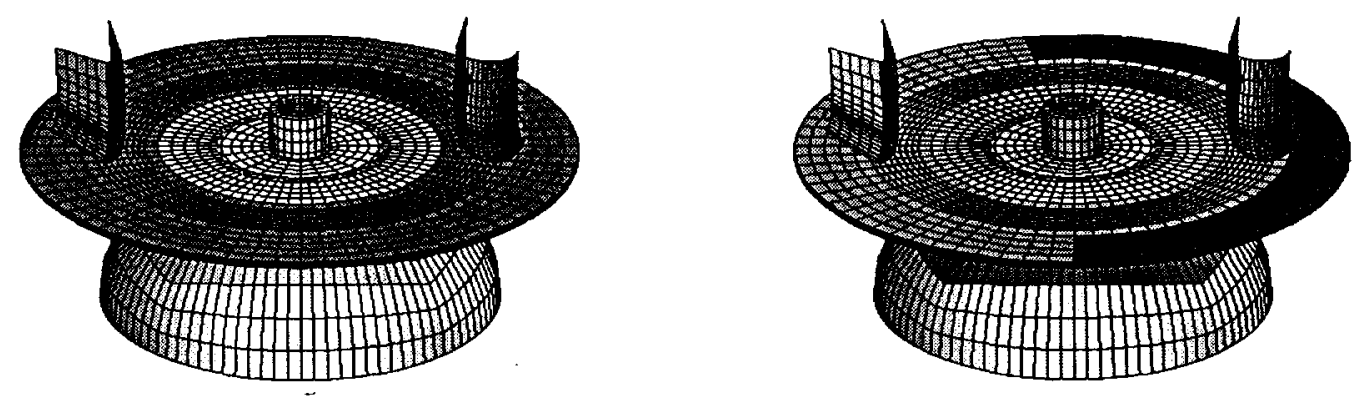

Figure 4. Four and eight-processor partitions for a spin forming problem. The model paritioned for four processors divides the plate into three concentric rings. The eightprocessor partition leaves the middle ring uncut.

\subsubsection{Parallel Automatic Contact}

For arbitrary contact defined with sliding interface types $12-14$, the analyst avoids the very time consuming task of defining the contact surfaces in the model. However, the parallel algorithm for these interface definitions is more expensive for two reasons. First, the search for contact is an expensive step, both on single processor computers and on parallel computers. Furthermore, for problems with considerable surface motion, the parallel versions of these algorithms accrue additional communication costs because the surfaces must be redistributed periodically among the processors. This surface redistribution load balances the contact calculations.

The efficiency of the parallel automatic contact can be improved by limiting the search domains with the DYNA3D keyword input options. Boxes may be used to delimit the search domains. Boxes save the computer time and storage required for the bucket sorts on the full mesh. This can save significant amounts of time for the large meshes designed for parallel computers. A further efficiency improvement is achieved with boxes by reducing the number of materials searched in each box using the material inclusion/exclusion options. 
The parallel automatic contact algorithm is currently under very extensive development and optimization. The largest effort underway now is to load balance the parallel automatic contact. Until this effort and several other new techniques to optimize the algorithm are more complete, it is recommended that data sets using automatic contact definitions be tested for performance before use in production simulations. ParaDyn developers would be grateful for any benchmark tests that demonstrate a need for improved optimization when using the automatic contact algorithm. In return we will provide insights on modeling with the algorithms that may provide better performance. We will also use the benchmarks to guide our algorithm development for optimizing the method, particularly for models with rapidly changing surface topologies, nested surfaces, and meshes with moving objects.

\subsubsection{Concluding Remarks on Parallel Contact}

For a complex mesh it may be beneficial to use both local and arbitrary contact algorithms and to provide multiple instances for each type of contact. An obvious advantage in doing this is that the regions on a mesh without any contact are not included in either the partitioning for local contact or the search domains for arbitrary contact.

For problems with a relatively small amount of interface surfaces compared to the volume of materials and with localized interfaces (types 1-3 and 5-10), the balance numbers provided by the partitioning software can be used for selecting the number of processors to use. Furthermore, the parallel efficiency may be determined by the size of the largest contact surface.

For efficient parallel arbitrary contact (automatic contact) it is very important to use DYNA3D input options to limit the search domains. This algorithm is currently undergoing extensive code development for parallel optimization.

\subsection{Boundary Conditions and Constraints}

Parallel versions of boundary conditions and constraints are treated both in the partitioning software as well as in ParaDyn. Because the partitioning software uses special processing on selected boundary conditions and constraints, it is important to know which boundary conditions and constraints are treated with partitioning and how this affects the overall partitioning. 
The following DYNA3D options are treated with the partitioning software $[4,10]$ :

Symmetry planes with failure

Nodal forces and follower forces

Nodal constraints

Tie-breaking shell slidelines

Tied node sets with failure

Rigid body joints

Shell-solid interfaces

Discrete springs, dampers and masses

One-dimensional slidelines.

These DYNA3D objects contain nodes and elements that must be assigned to a single processor rather than divided across more than one processor. Nodes that need to be kept together are assigned to Special Nodal Points (SNP) sets in the partitioning software. Associated with each of the SNP sets is a Special Element (SE) set. The SE set consists of all elements that contain one or more nodes in the corresponding SNP set. Each SNP set and its associated SE set must be fully contained in a processor. As a result, large SNP or SE sets can constrain a mesh partitioning and limit the number of processors that can be used for the problem. Reference [4] and the online version provide examples. 


\subsection{ANALYSIS WITH PARADYN}

An important consideration in our parallel algorithm development has been the requirement to provide automated tools for developing models for parallel computers. Automated tools have been developed for the new steps needed in either preprocessing the mesh or post-processing the binary plot databases. This chapter describes the ParaDyn software and how to use it.

\subsection{The ParaDyn Software Set}

The steps for running a ParaDyn simulation are shown in Figure 5.

\begin{tabular}{|c|c|c|}
\hline \multicolumn{3}{|c|}{ Step 1. Mesh Generation } \\
\hline \multicolumn{3}{|c|}{ Generate the mesh with special attention given to contact. } \\
\hline \multicolumn{3}{|c|}{ Step 2. Mesh Partitioning } \\
\hline \multicolumn{3}{|c|}{ Determine the optimal number of processors to use. } \\
\hline \multicolumn{3}{|c|}{ Step 3. ParaDynAnalysis } \\
\hline \multicolumn{3}{|c|}{ Run the ParaDyn analysis. } \\
\hline \multicolumn{3}{|l|}{ Step 4. Visualization } \\
\hline $\begin{array}{c}\text { Display results on a paral- } \\
\text { lel computer. }\end{array}$ & OR & $\begin{array}{c}\text { Display results on a work- } \\
\text { station. }\end{array}$ \\
\hline
\end{tabular}

The new preprocessing step for mesh partitioning is automated with script files, DYNAPART and DYNAPARTAGAIN. These files and other new utilities for parallel simulations are summarized in Figure 6.

The ParaDyn program is usually run with a utility, such as MPIRUN or POE, that copies the executable to the processors on a parallel computer. Most large simulations are run using batch processing software supplied by the Computer Center. Script files are often made available in public file systems to automate batch runs. 
There is a new step in post-processing of the binary databases with either state data or time history data. This step involves combining the plot database families generated on each processor into a single plot database family that can be viewed with GRIZ4 or THUG. The two utilities, XMILICS and COMBINETHS, are used for combining the state and time history plot databases, respectively. GRIZ4 and THUG can be used to visualize the results on either a single node on the parallel computer or on a workstation. Often for larger problems, the combined databases are transported with FTP to a workstation for visualization.

The software products used for ParaDyn simulations are summarized in Figure 6. The next sections describe these products in detail. The final section summarizes the steps for using ParaDyn software and can be used as a handy reference once the details of each step are understood.

\begin{tabular}{|c|c|c|c|}
\hline Task & Software & Input & Output \\
\hline \hline Mesh Partitioning & $\begin{array}{c}\text { DYNAPART } \\
\text { DYNAPARTAGAIN }\end{array}$ & DYNA3D input & $\begin{array}{c}\text { Partition map, plot file for } \\
\text { the partitioned mesh }\end{array}$ \\
\hline Parallel DYNA3D & ParaDyn & $\begin{array}{c}\text { DYNA3D input } \\
\text { Partition map }\end{array}$ & $\begin{array}{c}\text { Families of files: plot/time } \\
\text { history output, restart, ... }\end{array}$ \\
\hline $\begin{array}{c}\text { File combiners: } \\
\text { state and time his- } \\
\text { tory data }\end{array}$ & XMILICS & $\begin{array}{c}\text { Families by } \\
\text { processor of } \\
\text { state or time } \\
\text { history data }\end{array}$ & $\begin{array}{c}\text { One family for state or } \\
\text { time history data }\end{array}$ \\
\hline Visualization & GRIZ4, THUG & $\begin{array}{c}\text { State, time his- } \\
\text { tory databases }\end{array}$ & $\begin{array}{c}\text { Screen display, RGB out- } \\
\text { put, .... }\end{array}$ \\
\hline
\end{tabular}

Figure 6. ParaDyn Software Products.

\subsection{PATH Variable for ParaDyn}

The directory containing ParaDyn software must be included in the list in the PATH variable. The following SET PATH command added to the .cshrc file places the directory containing ParaDyn software products into the path variable.

set path $=($ usr/apps/bin/mdg \$path) 


\subsection{Partitioning a Model}

Before running ParaDyn, the model (including mesh, contact surfaces, constraints, and boundary conditions) must be partitioned using DYNAPART. It is often desirable to partition the model several times in order to select the number of processors to use for the model. The partitioning software provides statistics for guiding this selection process. DYNAPART and a related tool, DYNAPARTAGAIN, are script files which run several utilities for mesh and contact surface partitioning. The statistical output is written into the logfile generated when running these script files. Two utilties run within the scripts, METIS and PFGEN, produce the statistics for the partitioning of the model.

Since the partitioning software generates a number of intermediate files in the current working directory, running the partitioning scripts from a subdirectory is recommended. This conveniently groups the files containing the results generated for each mesh that is partitioned. The DYNAPART script is used the first time a mesh is partitioned. If the files from the first partitioning of a mesh are not destroyed, DYNAPARTAGAIN can be used for subsequent partitioning of the same mesh for a different number of processors. DYNAPARTAGAIN uses results saved from the DYNAPART execution and consequently, can skip over some of the time consuming steps in the DYNAPART script. It is very advisable to use DYNAPARTAGAIN when repartitioning a large model.

As a rule of thumb, an initial estimate for the optimal number of processors to use is roughly 2000 elements per processor. This estimate may be inadequate if contact surfaces, boundary conditions, or nodal constraints are restricting the partitioning (See discussions in sections 2.4 and 2.5.)

The execute lines for DYNAPART and DYNAPARTAGAIN are identical. The first argument is the name of the DYNA3D input file and the second argument is the number of processors requested for the mesh partitioning.

\section{dynapart infile $n p$}

dynapartagain infile $n p$

The output from the mesh partitioning is a file that is referred to as the partition file. The name of the partition file is infile.np. For the $d 3$ sampl input file for DYNA3D, the file name for a fourprocessor partition is 23 samp 1.4. The first several lines in the partition file contains partitioning statistics for the problem and the remainder of the file contains the list of element and node assignments to each processor. This file can be viewed with a text editor. 
The partitioning scripts also generate a plot database that can be used to visualize the subdomains of the partitioned model. The root name for the family of files is parplt. GRIZ4 can be used to visualize this database. The result is a coloring of the mesh subdomains by processor and a corresponding map of color values associated with the processors.

Caution: The database family, parplt, is overwritten when the partitioning scripts are run again in the same directory. The database can be saved by renaming it before running the next partitioning of the model.

The parallel efficiency for a particular mesh partitioning can be evaluated from statistics provided on the screen or in a log file generated by the partitioning scripts. The METIS software calculates and prints statistics for evaluating the quality of the partitioning. This output is listed as the Balance number from METIS. The best balance is obtained when this number is as close as possible to a value of 1.000 . Other statistics indicating the quality of the partitioning are printed by another tool, PFGEN, which is run by the scripts. The PFGEN output lists statistics about the shared-node communication resulting from the partition. The Uniformity number output by PFGEN measures the spread and the quantity of the communication among the processors. Again the best value for the communication uniformity is a number close to 1.0 . Figure 7 is an excerpt from the screen output containing these statistics.

The balance and uniformity numbers do not provide a measure of the relative amount of communication occuring within any given processor. These statistics can be obtained by inspecting the partition file. The total number of nodes in a processor and the number of shared nodes in a processor are listed in the first lines of the partition file. See Figure 8. For an efficient calculation it is useful to keep the ratio of the number of shared nodes to the total number of nodes as small as possible. Ideally this ratio would be ten percent or less. 


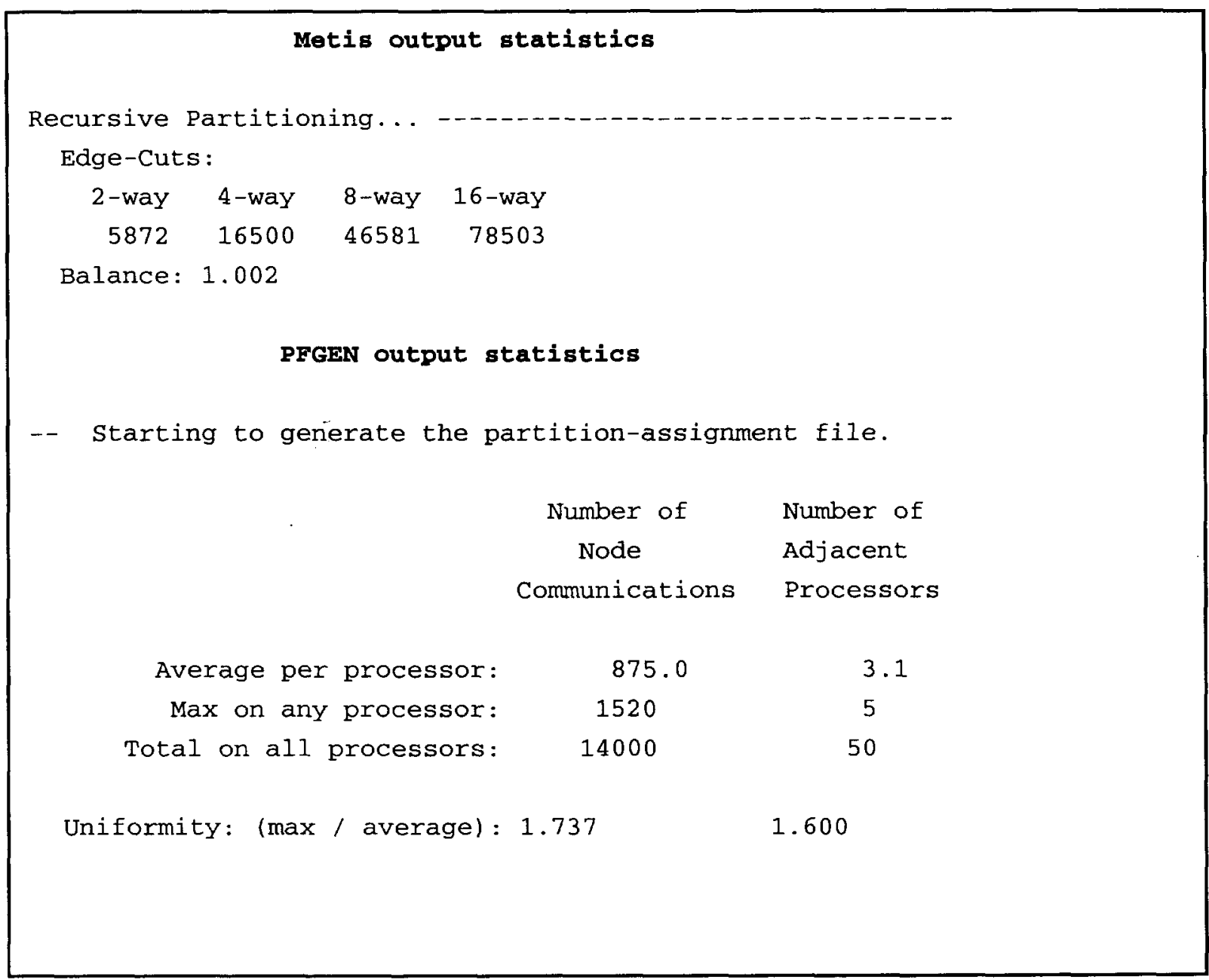

Figure 7. An excerpt from the screen containing Metis and PFGEN output statistics.

It may be convenient to save the screen output from the scripts into a file while also viewing the results. The saved log file can be used to inspect the statistics as well as error messages generated when the DYNAPART scripts are run. The UNIX TEE command can be used to do this as follows:

dynapart infile $n p \mid \&$ tee infile.log

The details of the nodal communication (rather than averages) are listed in the first few lines of the partition file, infile.np. These can be conveniently examined with the UNIX HEAD command.

head -20 infile.np 
The results of this command are shown in Figure 9.

\section{Example: Finding an optimal number of processors}

This example illustrates the use of the mesh partitioning tools to find the optimal number of processors to use for the first example problem included with the DYNA3D program.

Step 1. Set up a directory and partition the problem for two-processors.

The name of the input file is $d 3$ sampl. The directory for the partitioning is named partition.

\section{mkdir partition \\ cd partition \\ In -s ../d3samp1 d3samp1}

The UNIX utility LN links the $d 3$ samp1 file in a different directory from the current directory (partition) without making a copy. This saves disk space when when working with large files. It is also convenient to use LN when making runs using the same input file and varying the number of processors. This is the case when studying the scaling and efficiency for a model.

Execute DYNAPART the first time the mesh is partitioned.

\section{dynapart d3samp1 2}

The output files from this partitioning are $d 3$ samp 1.2 and the plot database, parplt. Next, visualize the partitioned mesh with GRIZ4.

$$
\text { griz4s -i parplt }
$$

Step 2. Look at the statistics from the DYNAPART interactive session.

The statistics from PMETIS and PFGEN are shown in the excerpt from the log file in Figure 9. 


\section{Running PMETIS}

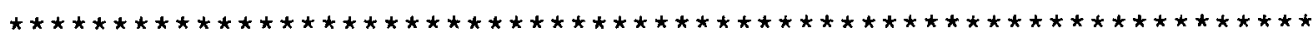

METIS 2.0 Copyright 1995, Regents of the University of Minnesota

Graph Information -

Graph: d3samp1.grfall, Size: 972, 19062, Parts: 2, Cto: 100

Options: SHEM, BGKLR, GGPKL, Rec-Partition

Recursive Partitioning....

Edge-Cuts :

2-way

360

Balance: 1.000

\section{Running PFGEN}

-- Starting to generate the partition-assignment file.

$\begin{array}{cl}\text { Number of } & \text { Number of } \\ \text { Node } & \text { Adjacent } \\ \text { Communications } & \text { Processors }\end{array}$

Average per processor:

Max on any processor:

Total on all processors:

Uniformity: (max / average):

++ Finished generating the partition-assignment file.
1.0

1

742
1.000
1.000

Figure 8. Partitioning statistics for a two-processor partition of the example mesh

Both of these statistics, the Metis Balance number and the Uniformity number written by PFGEN, suggest that the problem may be efficient if more processors are used for the partitioning of the model. 
The following are the detailed statistics for the shared nodal data obtained by running the HEAD command on the partition file, $d 3$ samp1.2. Notice that only 37 if the 486 nodes in each processor are shared.

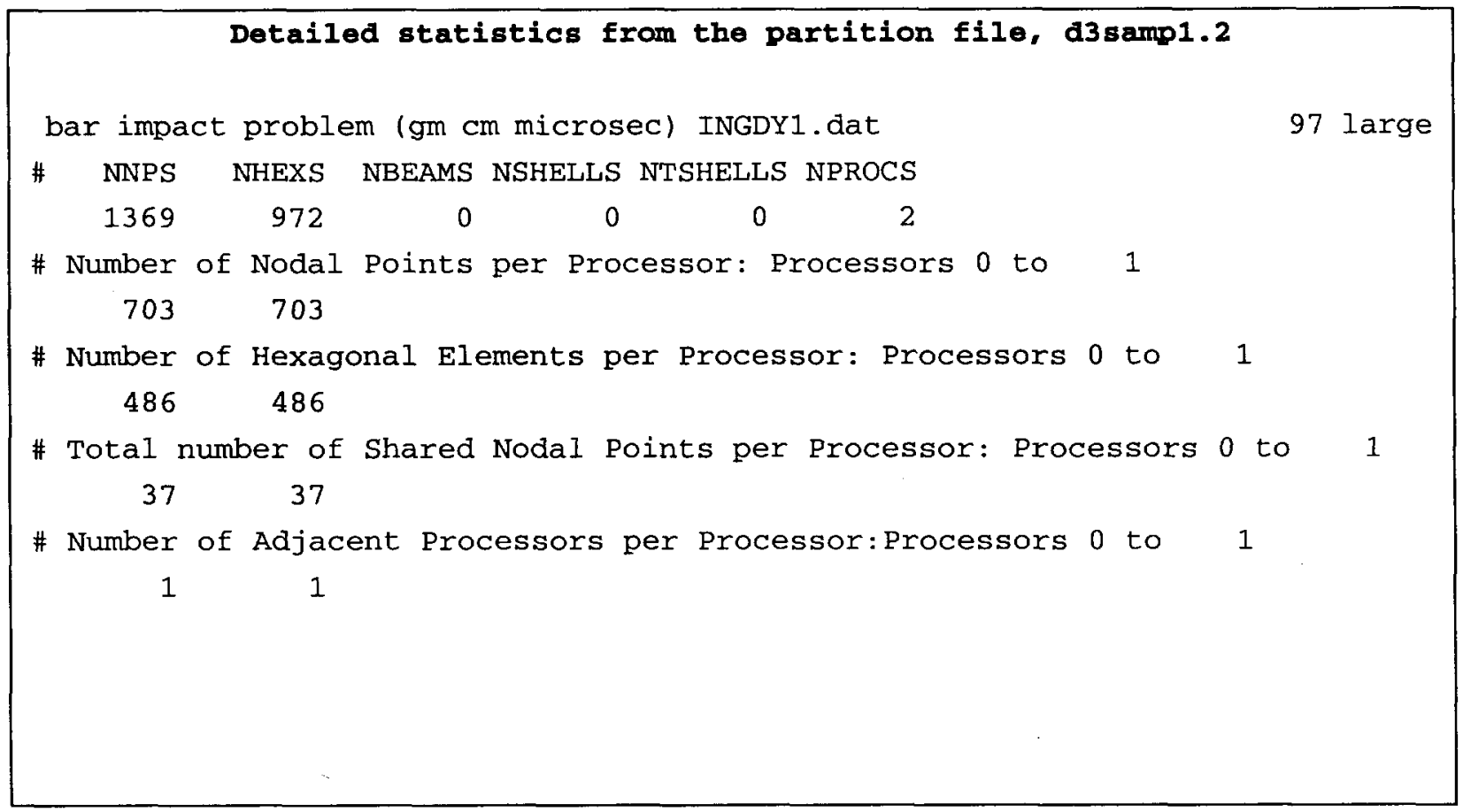

Figure 9. Total number of nodes, elements and shared nodes by processor for the two-processor partition

Step 3. Use DYNAPARTAGAIN to partition the model for four processors.

\section{dynapartagain d3samp1 4}

The partition file for this step is 13 samp1.4 and the plot database for the partitioned mesh overwrites the plot database for the previous partitioning.

The balance number from METIS for this partition is 1.000 indicating an even distribution of the computing work and communication among the four processors. The uniformity statistic from PFGEN shows that some processors have more communication. An inspection of the partition file, d3samp1.4, shows two of the processors engage in twice as much communication as the other two. The communicated nodes represent roughly $10 \%$ and $20 \%$ of the total number of nodes in the individual processors. These results are shown in Figure 10. 


\section{Partitioning d3sampl for 4 processors}

Running PFGEN

-- starting to generate the partition-assignment file.

$\begin{array}{cl}\begin{array}{cl}\text { Number of } \\ \text { Node }\end{array} & \begin{array}{l}\text { Number of } \\ \text { Adjacent }\end{array} \\ \text { Communications } & \text { Processors }\end{array}$

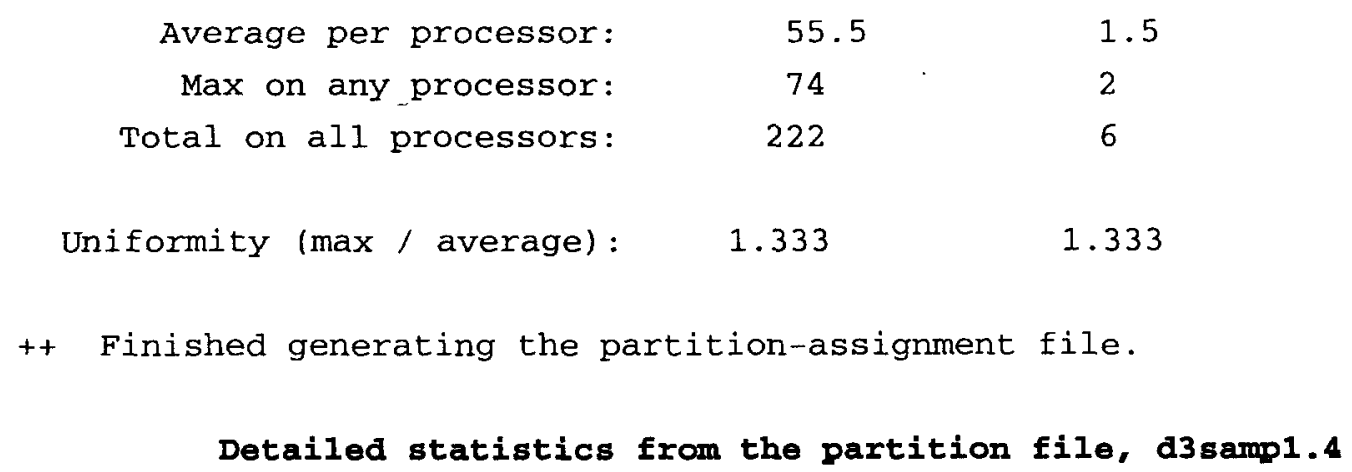

Figure 10. Total number of nodes, elements and shared nodes by processor for the four-processor partition

Step 4. Use DYNAPARTAGAIN to partition the mesh for eight processors. This partitioning is most likely to be poor based on the four-processor partition. The results shown below verify this.

\section{dynapartagain d3samp1 8}


The METIS balance statistic is 1.004 when the model is partitioned for 8 processors. Nevertheless, an inspection of the partition file shows that the number of shared nodes per processor varies between $22 \%$ and $40 \%$ of the total number of nodes in each processor. This is an unacceptably high amount of communication. The statistics in the partition file are shown below.

We can conclude from the partitioning for two, four and eight processors that the optimal number of processors to use for this problem is four.

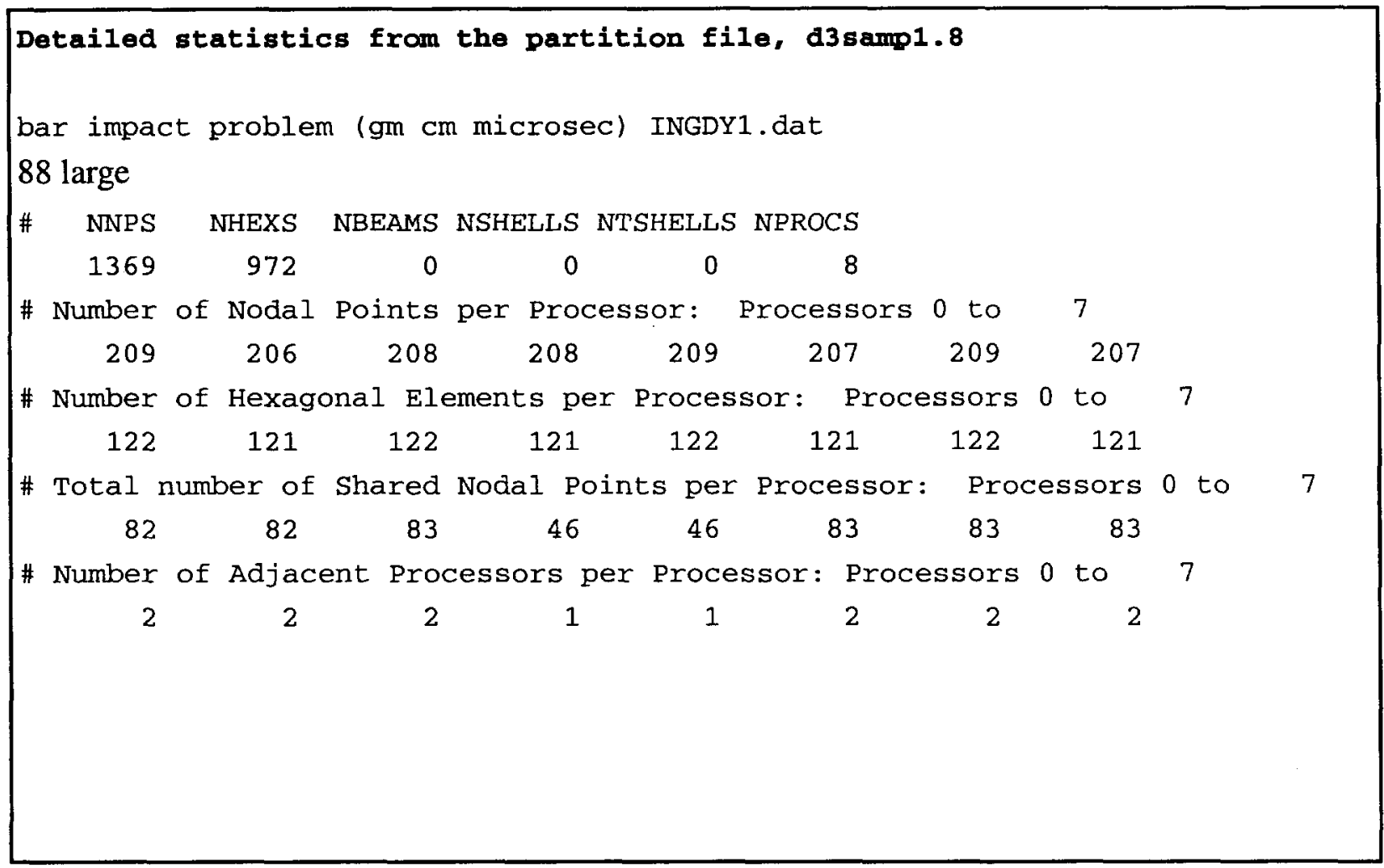

Figure 11. Total number of nodes, elements and shared nodes by processor for the eight-processor partition

\subsection{File Name Sequences}

The names of restart files and plot databases generated in a ParaDyn run are different from those for a DYNA3D simulation.The names are lengthened to include a processor number in the string for the root name for the database. More generally, each processor generates a set of files equivalent to a DYNA3D run on a single processor. The files are distinguished from the standard set in a DYNA3D run by a shortening of the root name to three characters and the inclusion of a processor number in the string for the name. For a problem running on 1000 or more processors, the length of the string appended to the root name is adjusted to accommodate the number of digits contained 
in the number of processors used. For example, for 1024 processors, four digits are added to the root name. Similarly for 10240 processors, five digits are added to the root name.The plot database names shown in Figure 12 are the default names generated for MILI state database files for a problem with less than 1000 processors.

\begin{tabular}{|c|c|c|c|}
\hline \multicolumn{4}{|c|}{ 1. State and time history databases: Root names $m_{-} p$ and tht } \\
\hline $\mathbf{P}_{\mathbf{0}}$ & $\mathbf{P}_{\mathbf{1}}$ & $\mathbf{P}_{\mathbf{2}}$ & $\mathbf{P}_{3}$ \\
\hline $\begin{array}{c}\text { m_p000A } \\
\text { m_p000 }\end{array}$ & $\begin{array}{c}\text { m_p001A } \\
\text { m_p001 }\end{array}$ & $\begin{array}{c}\text { m_p002A } \\
\text { m_p002 }\end{array}$ & $\begin{array}{c}\text { m_p003A } \\
\text { m_p003 }\end{array}$ \\
\hline m_p00001. & m_p00101 & m_p00201 & m_p00301 \\
\hline m_p00002 & m_p00102 & m_p00202 & m_p00302 \\
\hline$\ldots$ & $\ldots$ & $\cdots$ & $\ldots$ \\
\hline$\cdots$ & $\ldots$ & $\ldots$ & $\ldots$ \\
\hline m_p00099 & m_p00199 & m_p00299 & m_p00399 \\
\hline \multicolumn{4}{|c|}{ 2. Restart database names: Root names dmp } \\
\hline $\mathbf{P}_{\mathbf{0}}$ & $\mathbf{P}_{1}$ & $\mathbf{P}_{2}$ & $\mathbf{P}_{\mathbf{3}}$ \\
\hline dmp000p01 & dmp001p01 & dmp002p01 & dmp003p01 \\
\hline dmp000p02 & dmp001p02 & dmp002p02 & dmp003p02 \\
\hline dmp000p03 & dmp001p03 & dmp002p03 & dmp003p03 \\
\hline \multicolumn{4}{|c|}{ 3. Text output file names } \\
\hline $\mathbf{P}_{\mathbf{0}}$ & $\mathbf{P}_{1}$ & $\mathbf{P}_{2}$ & $\mathbf{P}_{\mathbf{3}}$ \\
\hline d3hsp & d3hsp001 & $\mathrm{d} 3 \mathrm{hsp} 002$ & d3hsp003 \\
\hline frc000 & frc001 & frc002 & frc003 \\
\hline
\end{tabular}

\subsection{ParaDyn Command Lines}

The partition file must be copied into a file named partfile before executing ParaDyn. Do not remove the original partition file. You can also use a soft link if the files are big. This is explained in the examples. The partition file is used with the post-processing software. 
The ParaDyn execute line arguments are identical to the DYNA3D execute line. The restarts for ParaDyn likewise use the same execute line as those used in executing a DYNA3D restart. The command lines for a ParaDyn initial run and a restart execution are illustrated in the following two examples.

$$
\begin{aligned}
& \text { paradyn } \mathbf{i}=\text { infile } \mathbf{l}=\text { filelength } \\
& \text { paradyn } \mathbf{i}=\text { restart } \mathbf{r}=\text { dumpfile } \mathbf{l}=\text { filelength } \\
& \text { paradyn } \mathbf{i}=\text { infile } \mathbf{q}=\text { nseconds } \\
& \text { paradyn } \mathbf{c}=\text { lastdump } \mathbf{q}=\text { nseconds }
\end{aligned}
$$

The first set of execute lines uses the standard input lines for restarts described in the DYNA3D manual. The second set of execute lines illustrates a restart method which starts from the last successfully written restart file.

Caution: The standard output files, $(\mathrm{d} 3 \mathrm{hsp}, \mathrm{d} 3 \mathrm{hsp} 0001, \ldots)$ are overwritten when a subsequent run is made in the same directory. It is generally a good practice to at least save the file $d 3 h s p$ into a different name between restarts of the problem. This output file, $d 3 h s p$, usually has results that are of value at the end of a long sequence of restarts. For instance, filenames, input options, and dynamic relaxation results are written into the file $d 3 h s p$.

The $c=$ option specifies the name of a file which is always lastdump. ParaDyn generated the lastdump file at the end of the most recent run made in the directory. ParaDyn writes one line in the lastdump file. This line conains the name of the dump restart file which has the last successfully written state data. This is a very important option to use on some parallel computers, particularly the ASCI Blue computer at LLNL.

Notice that it is not necessary to specify an input file for a restart execution. If the input file is not specified, all input options will remain the same as those specified in the preceding run. These values from the previous run are stored in the restart dump file and read in during the input phase of the restart run.

The $\mathbf{q}$ option provides a capability for terminating a problem on the ASCI Blue Pacific system. The value, in seconds, specifies the number of seconds (by the wall clock) to run the ParaDyn simulation before ParaDyn stops itself with a normal termination. This option is often used to allow 
the analyst to specify a wall-clock termination time slightly shorter than the batch time he selects. The extra time allows for final file writes and close operations for the database families and other output files.

The $\mathbf{l}=$ option provides a method for increasing the maximum file length for each member of a database family of plot files. The size specified is in units of Megabytes. Caution: If the file length option is used on the initial ParaDyn run, it must be used on all subsequent restarts.

\section{Example: Execute lines for ParaDyn}

Consider an initial ParaDyn run using the DYNA3D input file, $d 3$ sampland a restart run using an input data file, irestart. Following the naming convention in Figure 12, the initial run and restart run are executed with the following ParaDyn input arguments.

$$
\begin{aligned}
& \text { paradyn } i=d 3 \operatorname{samp} 1 \mathrm{l}=10 \\
& \text { paradyn } i=\text { =irestart } r=d m p 000 p 01 \mathrm{l}=10
\end{aligned}
$$

Notice, the file lenth is specified on both the initial run and on the restart.

This next example illustrates the use of the $\mathbf{q}=$ termination option.

$$
\begin{aligned}
& \text { paradyn } i=d 3 \operatorname{samp} 1 q=3000 l=100 \\
& \text { paradyn } c=\text { lastdump } q=3000 l=100
\end{aligned}
$$

This problem will be submitted to batch to run for one hour ( 3600 seconds) and ten minutes before the end of the run (afer 3000 seconds) ParaDyn will call the termination routine to close files and exit. The maximum length of the files in the plot databases is set to 100 Megabytes. The name of the restart file is the name listed on the first line of the file named lastdump.

\subsubsection{ParaDyn Run Interactively}

ParaDyn can be run interactively using the POE utility on an ASCI Blue computer at LLNL. This system has four processors per node and five hundred or more nodes. The POE utility runs ParaDyn with an input specifying the number of nodes and optionally, the total number of processors. The options for specifying the number of nodes and processors are inserted between the ParaDyn command and the execute line options for ParaDyn. If only one processor per node is being used, then it is sufficent to simply specify the number of nodes as follows: 
poe paradyn -nodes numnodes executeline

More than one processor per node can be used by specifying the number of nodes with -nodes and the total number of processors with -procs.

poe paradyn -nodes numnodes -procs np executeline

Example: Interactive ParaDyn run on the ASCI Blue Pacific

The following are command lines for executing ParaDyn with the DYNA3D test case 1 as an input file and using either four or five processors.

poe paradyn -nodes 4 -procs $1 i=d 3 s a m p 1$
poe paradyn -nodes 1 -procs $4 i=d 3 \operatorname{samp1} 4$
poe paradyn -nodes 2 -procs $5 i=d 3 \operatorname{samp1} 4$

The first example above will run using one processor on each of four nodes. The second example will run with four processors on one node. The third example will run on two nodes using five of the eight processors.

ParaDyn can be run interactively using the MPIRUN utility on an Origin 2000 computer. The MPIRUN utility runs ParaDyn for $n p$ processors as follows.

mpirun -np np paradyn dyna3d execute line

Example: Interactive ParaDyn run on the Origin 2000

The following is the command line for executing ParaDyn with the DYNA3D test case 1 as an input file and using 4 processors.

$$
\text { mpirun -np } 4 \text { paradyn i=d3samp1 }
$$

\subsubsection{ParaDyn Run with Batch}

ParaDyn production simulations are run under the DPCS batch processing system on the ASCI Blue computers at LLNL. To set up a problem for batch, a script file is prepared and then submitted to the batch system with the PSUB utility. Lines included in a typical script file for a ParaDyn simulation are shown in Figure 13. 
The script file is submitted to batch for processing using the PSUB utility.

psub scriptfile

Use the PSTAT utility for interrogating the status of runs submitted with PSUB. A status of RUN indicates the job is running on the parallel computer. Type SPJSTAT to further check on the status of the run after PSTAT indicates it is in a RUN state.The online MAN pages can be viewed to study other options provided by the PSUB and PSTAT utilities.

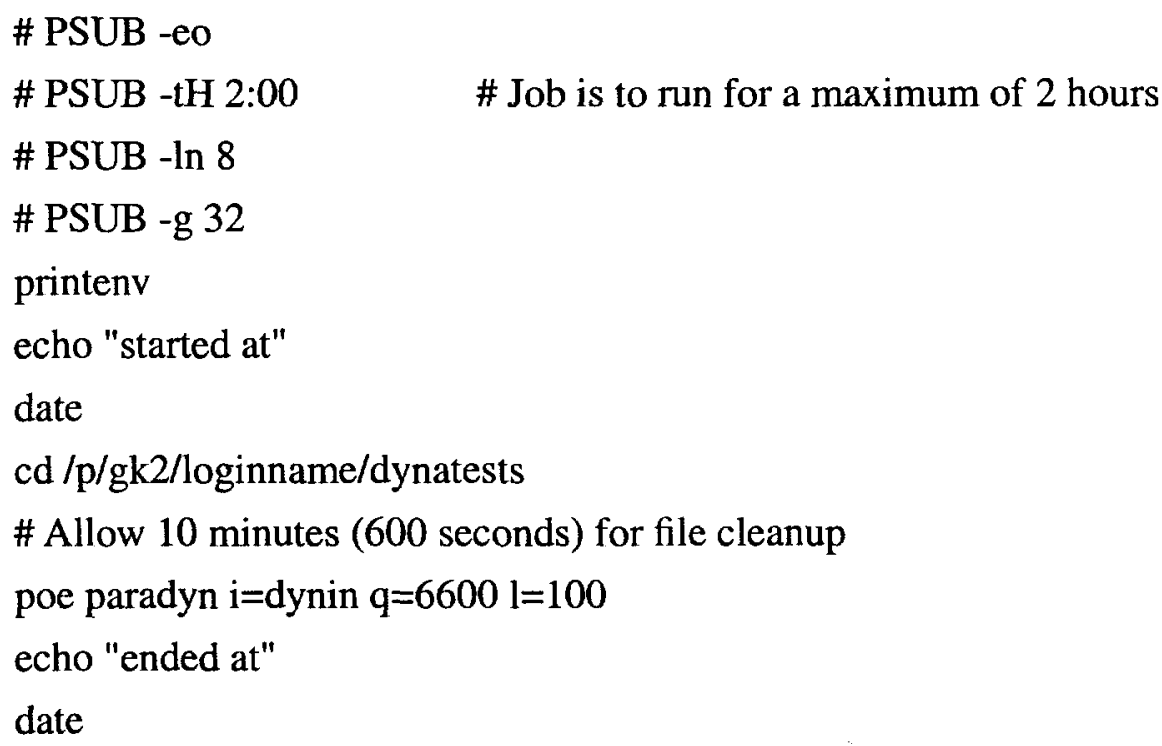

Figure 13. A typical batch script file for a ParaDyn simulation

\subsection{Visualizing ParaDyn Results}

There are two choices for post-processing the state database output from a ParaDyn analysis. First the database families from the processors must be combined with the utility XMILICS. Then the results may be viewed on the parallel computer with GRIZ4. Alternatively, the combined database families may be transported to a workstation and viewed with GRIZ4. 
There are some tradeoffs to be made in selecting either of these choices. For large problems particularly, the rendering and the display of a frame sent over a network to a workstation can be as long as twenty or thirty minutes. In this case better interactivity is possible by using FTP to send the combined database over to a local workstation. The additional resources needed for this improved interactivity are twice the disk space on the parallel computer, a workstation with highspeed graphics capabilities, and enough disk space on the workstation for the full database. Even with this additional hardware, the viewing of results is delayed by the time it takes to FTP the databases to the workstation. In the future, parallel rendering and visualization tools will become available that will eliminate some of these delays.

\subsubsection{MILI Database Files}

MILI plot databases are new. The format for the MILI databases provide a considerable amount of flexibility for designing material templates that display results specific to each material type as well as the flexibility for selecting which state data fields should be included in a database generated by a parallel analysis. Equally as important, the menus in GRIZ4 will reflect those data fields, result fields, and names which have been written into the MILI files. GRIZ4 must be used for displaying MILI plot databases.

Recent code development in both ParaDyn and DYNA3D require the use of the MILI database format. In addition, the MILI databases have been extensively tested in large benchmarks and in production runs at both Livermore and Los Alamos. For these reasons, we encourage their use. The old TAURUS database format used with ParaDyn is described in Section 4.5.

The use of GRIZ4 is also strongly recommended because of its expanded capabilities to handle the menu- generation for MILI databases. In the future, Mili database formats will be developed to provide many new features for material models, to display new results (not currently in TAURUS databases), to highlight boundary conditions, and to provide flexibility for limiting the size of parallel databases.

The MILI database software was released with ParaDyn version 1.01 and will be the default database type in future versions of ParaDyn. In addition, it is possible to select one or both database formats in the keyword control options input section of the input file. The keyword specification is:

mili_plot miliflag

endfree 
The value of the flag is set to 1 to select the MILI database format and is set to 0 to deselect the database type.

Example: Specify MILI for the plot database format in a ParaDyn analysis.

Add the following keyword input to the keyword control options section of the input file.

mili_plot 1

endfree

\subsubsection{Combining Parallel Databases}

The utility XMILICS combines the state database from a ParaDyn run. The combined databases can then be viewed with GRIZ4. The execute line for XMILICS is

\section{xmilics -i infileroot $\mathbf{- 0}$ outfileroot $-\mathbf{c}$ partfile}

The first argument is the root name for the families of state databases and the second argument is the root name for the output files with the combined databases. The third argument is the name of the partition file.

The utility COMBINETHS combines the time history database from a ParaDyn run. The combined time history database can then be viewed with the THUG uility. The execute line for COMBINETHS is

combineths infileroot outfileroot partfile 00

The first argument is the root name for the families of time history databases and the second argument is the root name for the output files with the combined databases. The last two arguments must both be 0 .

Example: XMILICS and COMBINETHS execute lines

xmilics -i m_p -o mout -c partfile

combineths plt oplt partfile 00

See Figure 14 for the list of output files generated with each combining utility. 
XMIIICS output files

moutA, mout 00, mout01, mout02, mout $03, \ldots .$.

COMBINETHS output files

otht00, otht 01 , otht $02, \ldots$.

Figure 14. The names of files in output databases from XMILICS and COMBINETHS.

On the workstation or the parallel computer, these databases are viewed with the GRIZ4 and THUG utilities.

$$
\begin{aligned}
& \text { griz4s -i mout } \\
& \text { thug -i otht }
\end{aligned}
$$

\subsection{Summarized Steps for Using ParaDyn}

Step 1. Set the PATH variable for the ParaDyn software.

This line is added in the .cshrc file the first time the software is used.

set path=(/usr/apps/bin/mdg \$path)

Step 2. Partition the mesh.

Make a directory for partitioning. Find the optimal number of processors for the run.

mkdir partition

cd partition

In -s ../d3samp1 d3samp1

dynapart d3samp1 2

dynapart d3samp1 4

dynapart d3samp1 8

mv d3samp1.2 d3samp1.4 ../

cd ..

Step 3. Execute ParaDyn initial and restart runs

Make a directory for the simulation. Link the input and partition files into the directory.

mkdir rundir 


\section{cd rundir \\ In -s ../d3samp1 d3samp1 \\ In -s ../d3samp1.4 partfile \\ paradyn $\mathrm{i}=\mathrm{d} 3$ samp1}

Save the $d 3 h s p$ output file between restarts. Input new options with the file irestart.

mv d3hsp hsprun1

paradyn i=irestart $d=d m p 000 p 01$

mv d3hsp hsprun2

Use the lastdump file to get the name of the last successfully written restart dump file. There are no resets of the input options for the next restart.

paradyn c=lastdump

Step 4. Visualize the results.

Combine the state database and the time history databases.

xmilics -i m_p -o mout -c partfile

combineths tht otht d3samp1.4 00

Move the combined state and time history databases to the workstation, if desired. View the results on either computer.

$$
\begin{aligned}
& \text { griz4s -i mout } \\
& \text { thug -i otht }
\end{aligned}
$$




\subsection{INPUT FOR PARALLEL SIMULATIONS}

This chapter describes data input and special instructions used for parallel simulations only. Instructions for running multiple ParaDyn analyses and special instructions for post-processing results written to text files are described here.

\subsection{Static Initialization and Dynamic Analysis}

Dynamic relaxation and stress initialization techniques are described in the DYNA3D manual. The following example shows one method for initializing a problem with a load, generating the stresses from a relaxation procedure, and starting a time dependent simulation. Three runs are used for this procedure.

Step 1. Run the relaxation step first.

This feature is activated by a flag on the load curve definition cards in the standard DYNA3D input.

$$
\text { paradyn i=input1 }
$$

This creates two dump files for each processor. For processor zero, they are $d m p 000 p 01$ and dmp000p02.

Step 2. This step generates a stress output file.

Restarting the previous run for one time step to generate the stress file.

\section{paradyn $i=$ restart $r=d m p 000 p 02$}

This run creates the stress file, $d 3 s p p p$, where $p p p$ is the processor number.

Step 3. This steps runs a dynamic analysis using the stress file from the static initialization.

paradyn $i=$ input $2 \mathrm{n}=\mathrm{d} 3 \mathrm{~s}$ 


\subsection{The NIKE-DYNA Link File}

The NIKE-DYNA link file is often used to start up a new run using stress results from a previous run on the same mesh. The details for this technique are described in section 2.19 of the DYNA3D manual. The first run to generate the stress file must include the following free format input to generate the output database with the stresses. The root name for the link file is specified on the following keyword input line.

nikefile stressfile

endfree

A ParaDyn execution with this input selecting the name, str, for the NIKE-DYNA link file will create database families for each processor labeled

str001, str002, ... strppp, ...

where $p p p$ is the processor number.

A second run uses the same input mesh but other parameters in the input, such as load curves, may be changed. (Although the control options can be changed, it is not a requirement, and the same data input file can be used for both runs.) The second run reads the stress databases, stores the stress data, and uses the deformed nodal coordinate values from the first run. Other parameters associated with the original run may also be set and are described in the section Keyword-Based Control Features of the DYNA3D manual.

\section{Example: Generate a NIKE-DYNA link file with ParaDyn}

Consider a run with an input file, $d 3 s t$, specifying the name stress on the nikefile keyword-input line. A second run will use the generated stress database by specifying the $\mathbf{m}=$ option on the execute line and new DYNA3D options in the input file, d3st1.

$$
\begin{aligned}
& \text { paradyn } i=d 3 s t \\
& \text { paradyn } i=d 3 s t 1 \mathrm{~m}=\text { =stress } g=g l t
\end{aligned}
$$

In the second run, the plot state database file family is given a new root name, glt. 


\subsection{Multiple Versions of Running Restart Files}

The following describes a new capability for generating multiple versions of a running restart dump file. The option is selected with a keyword input and optionally specifying the running restart file names on the ParaDyn execute line. The keyword input is

\section{numrrf nvers}

The integer value, nvers, specifies the number of different versions of the running restart file to save on disk. The running restart family names are incremented through a set of family members until nvers of them have been written. Once this limit is reached the next running restart over writes the first running restart file in the set. The examples below illustrate the cycling through of the names of the versions of the running restart files.

The number of cycles between the running restart dump files is selected as usual in the fifth data field, columns 36-40, of DYNA3D control card 6. The default names for the running restart file for ParaDyn are rsfnnnmm, where $n n n$ is the three-digit processor number and $m m$ is the familymember number. To select the name of the running restart file, use the $\mathbf{a}=$ option on the ParaDyn execute line.

\section{Example: Specify multiple versions of the running restart file}

Set up the input file to save three different versions of the running restart file.

\section{numrrf 3}

endfree

Suppose a problem is run for 100 cycles and the number of cycles between running restart dumps is 30 . The files generated for a 4-processor run will be:

$$
\begin{array}{ll}
\text { rsf00001 rsf00101 rsf00201 rsf00301 } & \text { (cycles 30) } \\
\text { rsf00002 rsf00102 rsf00202 rsf00302 } & \text { (cycles 60) } \\
\text { rsf00003 rsf00103 rsf00203 rsf00303 } & \text { (cycles 90) }
\end{array}
$$

Suppose this problem is run for another 150 cycles, then the running restart files will be 


$$
\begin{array}{ll}
\text { rsf00002 rsf00102 rsf00202 rsf00302 } & \text { (cycle 150) } \\
\text { rsf00001 rsf00101 rsf00201 rsf00301 } & \text { (cycle 120) } \\
\text { rsf00003 rsf00103 rsf00203 rsf00303 } & \text { (cycle 180) } \\
\text { rsf00001 rsf00101 rsf00201 rsf00301 } & \text { (cycle 210) } \\
\text { rsf00002 rsf00102 rsf00202 rsf00302 } & \text { (cycle 240) }
\end{array}
$$

In the above, the cycle $(30,60,90)$ files were over written by the cycle $(120,150,180)$ files, respectively. And finally, the cycle $(210,240)$ files over write the cycle $(120,150)$ files.

\section{Example: Select a new name for the running restart files}

Suppose instead in the previous example that the initial problem is restarted from cycle 60 and runs up to cycle 250. A new name can be selected for the versions of the running restart files as follows:

$$
\text { paradyn } i=i n r e s t, r=r s f 00002, a=n r f
$$

The files generated will be

$$
\begin{aligned}
& \text { nrf00001 nrf00101 nrf00201 nrf00301 (cycle 90) } \\
& \text { nrf00002 nrf00102 nrf00202 nrf00302 (cycle 120) } \\
& \text { nrf00003 nrf00103 nrf00203 nrf00303 (cycle 150) } \\
& \text { nrf00001 nrf00101 nrf00201 nrf00301 (cycle 180) } \\
& \text { nrf00002 nrf00102 nrf00202 nrf00302 (cycle 210) } \\
& \text { nrf00003 nrf00103 nrf00203 nrf00303 (cycle 240) }
\end{aligned}
$$

\subsection{Nodal Force Output}

The nodal force output is described in the keyword input and Section 4.47 of the DYNA3D input manual. Nodal force output is selected using the keyword input variable nfrout. The value of this keyword input variable is the total number of nodes that will be written into the output files.

\section{Output format}

For each time at which the nodal forces are desired, the following set of data are written:

Card $1(\mathrm{E} 12.5,1 \mathrm{x}, \mathrm{i} 8)$

Col. 1-12 Time at which forces have been computed E12.5 
Col.14-20 Number of nodes (nfrout)

Cards 2 to Card $1+$ nfrout (i8,3e12.7)

Col. 1-8 Node number I8

Col. 9-20 Force in the $\mathrm{x}$-direction E12.5

Col. 21-32 Force in the $y$-direction E12.5

Col. 33-44 Force in the z-direction E12.5

The name of the text output file containing the nodal force data is nodfrc for a DYNA3D run.

ParaDyn generates two kinds of text output files. The first output file (generated by processor zero only) contains the times at which the forces are output for the selected nodes. The name of this file is nfrctimes. The remaining output data, the node numbers and three components of the force, are written to a set of files generated by all of the processors. The files are named nfrcnnn, where $n n n$ is the three-digit processor number. By separating the data in this way, the following UNIX utilities can be used to combine the data into a single file, with the time steps for the output at the top, followed by the list of nodes and forces for each time step.

\section{cat nfre* $\mid$ sort $-\mathrm{n}->$ forceout}

\section{Example: Combine nodal force output from ParaDyn}

The following is an example in which two nodes, $(12,144)$, were selected and written at three different time steps. The nodes were merged using the files from the processors with the previous CAT and SORT utilites. The results are

$$
\begin{array}{rrrrr}
0.00000 \mathrm{E}+00 & 2 & & \\
0.44627 \mathrm{E}-06 & 2 & & & \\
0.89344 \mathrm{E}-06 & 2 & & & \\
12 & 0.00000 \mathrm{E}+00 & 0.00000 \mathrm{E}+00 & 0.00000 \mathrm{E}+00 \\
12 & 0.00000 \mathrm{E}+00 & 0.00000 \mathrm{E}+00 & 0.00000 \mathrm{E}+00 \\
12 & 0.00000 \mathrm{E}+00 & 0.00000 \mathrm{E}+00 & 0.00000 \mathrm{E}+00 \\
144 & 0.00000 \mathrm{E}+00 & 0.00000 \mathrm{E}+00 & -0.14000 \mathrm{E}-11 \\
144 & 0.00000 \mathrm{E}+00 & 0.00000 \mathrm{E}+00 & -0.14000 \mathrm{E}-11 \\
144 & 0.00000 \mathrm{E}+00 & 0.00000 \mathrm{E}+00 & -0.15000 \mathrm{E}-11
\end{array}
$$


To eliminate the time stamp data at the beginning of the file (and read it from the file nfrctimes instead in a post-processor), use the following UNIX utilities for combining the output.

\section{cat nfrc??? | sort - n - > forceout}

\subsection{TOPAZ3D Temperature Input}

The TAURUS database with nodal temperature data generated by the TOPAZ3D program can be input to a ParaDyn simulation. Input options are completely compatible with the DYNA3D/ TOPAZ3D temperature input options. The restriction for using this feature is that the same mesh must be used for both programs.

In the future we expect to develop a MILI database to link TOPAZ3D and DYNA3D/ParaDyn. The utility XMILICS currently has the capability to both combine and split MILI databases into a specified number of processors. This will allow a link between a parallel or single processor TOPAZ3D simulation and a ParaDyn simulation where the number of processors used for each code is not restricted to be the same.

\subsection{TAURUS Database Files}

Many of the new ParaDyn code developments require the use of the flexibile file formats provided by the MILI I/O library. For this reason, TAURUS databases are no longer generated by default in ParaDyn. Keyword input may be used to select TAURUS databases for those special circumstances where they may be needed. An example may be a restart from an older run ParaDyn run. The file name sequences for TAURUS databases is shown in Figure 15.

TAURUS plot databases are selected in the keyword input section of the DYNA3D/ParaDyn input file. Both MILI and TAURUS databases can be output in the same run. This capability is usually used by the code developers and not recommended for long analysis runs. The following two keywords select and deselect the TAURUS and MILI databases.

mili_plot miliflag

taurus_plot taurusflag

endfree

The value of either flags is set to 1 to select the database type and is set to 0 to deselect the database type. 


\begin{tabular}{|c|c|c|c|}
\hline \multicolumn{4}{|c|}{ 1. State and time history databases: Root names plt and tht } \\
\hline $\mathbf{P}_{\mathbf{0}}$ & $\mathbf{P}_{1}$ & $\mathbf{P}_{2}$ & $\mathbf{P}_{\mathbf{3}}$ \\
\hline plt000 & plt001 & plt002 & plt003 \\
\hline plt00001 & plt00101 & plt00201 & plt00301 \\
\hline plt00002 & plt00102 & plt00202 & plt00302 \\
\hline$\ldots$ & $\ldots$ & $\ldots$ & $\cdots$ \\
\hline$\ldots$ & $\ldots$ & $\ldots$ & $\ldots$ \\
\hline plt00099 & plt00199 & plt00299 & plt00399 \\
\hline
\end{tabular}

The PGRIZ utiltity is only available on the Livermore computer systems. This utility combines the TAURUS databases in memory when visualizing the plot files on the parallel computer. The execute line for the PGRIZ tool is

\section{pgriz -i filename -p partfile 00}

The first argument, filename, is the full name of the first file in the database family for processor 0 . The second argument, partfile, is the name of the partition file. The last two arguments are always set to zero.

\section{Example: PGRIZ execute line}

An example input line for the test case in the previous example is

$$
\text { pgriz -i plt -p d3samp1.4 }
$$

The utilities XMILICS and COMBINETHS combine the Taurus state and time history databases from a ParaDyn run. The state databases can be viewed with GRIZ4 and the time history databases with THUG.

The XMILICS execute lines is 


\section{xmilics -i infileroot $-\mathbf{0}$ outfileroot $-\mathbf{c}$ partfile}

The first and second arguments are the root names of the input TAURUS database and the combined output MILI database. The third argument is the name of the partition file.

The COMBINETHS execute line is

combineths infileroot outfileroot partfile $\mathbf{0 0}$

The first argument is the root name for the input families of time history databases and the second argument is the root name for the output family of files with the combined databases. The last two arguments must both be 0 .

\section{Example: XMILICS and COMBINETHS execute lines}

Consider the state and time history databases for the previous four-processor example. The root names for the plot databases and time history databases are plt and ths, respectively. The following execute lines will generate an output family of files with the root names oplt and oths, respectively.

xmilics -i m_p -o oplt -c d3samp1.4

combineths tht otht d3samp1.4 00

xMILICS output files

moutA, mout00, mout01, mout03, ...

COMBINETHS output files

otht00, otht01, otht02,...

Figure 16. The names of files in output databases from XMILICS and COMBINETHS.

On the workstation, these databases are viewed with the GRIZ4 and THUG utilities.

$$
\begin{aligned}
& \text { griz4s -i oplt00 } \\
& \text { thug -i otht }
\end{aligned}
$$




\subsection{FUTURE ENHANCEMENTS}

Parallel algorithm design is an integral part of the software development cycle for the DYNA3D/ ParaDyn software. The Design Phase includes the design of the approximate technique for the mechanics, the algorithm design for single processor computers, and the complementing design for parallel computers. Once a design is in place, frequently the software development proceeds with a serial (DYNA3D) implementation and testing. This then is followed by a parallel implementation and testing. The following features implemented in DYNA3D are future enhancements to the ParaDyn software.

1. Contact algorithms

LaGrange constraint forms of contact.

2. Rigid body algorithms

Deformable and rigid material switching algorithm.

3. Boundary conditions

Rigid nodal constraint sets.

4. New elements

Cohesive and delaminated elements.

There are two DYNA3D features which will not be implemented in parallel as follows:

1. Coupled codes

Madymo DYNA3D link capability.

2. Contact algorithms

Slide line type 11. This algorithm is an obsolete version of the SAND algorithm. The replacement algorithm in sliding interface type 14 is implemented in parallel. 


\section{REFERENCES}

1. ,Lin, Jerry I., "DYNA3D: A Nonlinear, Explicit, Dimensional Finite Element Code for Solid and Structural Mechanics-User Manual," Lawrence Livermore National Laboratory, Livermore, California, UCRL-MA-107254 Rev. 2, 1999.

2. Speck, D. E., Dovey, D. J., “GRIZ: Finite Element Analysis Results Visualization for Unstructured Grids-User Manual," Lawrence Livermore National Laboratory, Livermore, California, UCRL-MA-115696, 2000.

3. Speck, D. E., "MILI: A Mesh I/O Library-Programmer's Reference", Lawrence Livermore National Laboratory, Livermore, California, UCRL-MA (in publication) 2000.

4. Sherwood, Robert J., "DynaPart Auxiliary Documentation Files", Lawrence Livermore National Laboratory, Livermore, California, UCRL-ID-137888, 2000.

5. Schauer, D. A., Hoover, C.G., Kay, G. J., Lee, A.S., and De Groot, A.J., "Crashworthiness Simulations with DYNA3D", Paper No. 961249, Transportation Research Board, 1996.

6. Karypis, G. and Kumar, V., "METIS 3.0: Unstructured Graph Partitioning and Sparse Matrix Ordering System," University of Minnesota, Department of Computer Science, 1997. See also http://www-users.cs.umn.edu/ karypis/metis/main.shtml.

7. Karypis, G. and Kumar, V., "A Fast and High Quality Multilevel Scheme for Partitioning Irregular Graphs," SIAM Journal on Scientific Computing, 1998. A short version appears in Intl. Conf. on Parallel Processing, 1995.

8. Karypis, G. and Kumar, V., "Multilevel k-way Partitioning Scheme for Irregular Graphs," Journal of Parallel and Distributed Computing, 1997.

9. Hendrickson, B. and Leland, R., "An Improved Spectral Graph Partitioning Algorithm for Mapping Parallel Computations," Sandia National Laboratory Report Number SAND92$1460,1992$.

10. Hoover, C.G., Badders, D. C., De Groot, A.J., and Sherwood, R. J., "Parallel Algorithm Research for Solid Mechanics Applications Using Finite Element Analysis," Lawrence Livermore National Laboratory, Livermore, California, UCRL-ID-129202, 1997. 